\title{
A Numerical Scheme for Non-Newtonian Fluids and Plastic Solids under the GPR Model
}

\author{
Haran Jackson ${ }^{\mathrm{a}, * *}$, Nikos Nikiforakis ${ }^{\mathrm{a}}$ \\ ${ }^{a}$ Cavendish Laboratory, Department of Physics, Cambridge University, UK
}

\begin{abstract}
A method for modeling non-Newtonian fluids (dilatants and pseudoplastics) by a power law under the Godunov-Peshkov-Romenski model is presented, along with a new numerical scheme for solving this system. The scheme is also modified to solve the corresponding system for power-law elastoplastic solids.

The scheme is based on a temporal operator splitting, with the homogeneous system solved using a finite volume method based on a WENO reconstruction, and the temporal ODEs solved using an analytical approximate solution. The method is found to perform favorably against problems with known exact solutions, and numerical solutions published in the open literature. It is simple to implement, and to the best of the authors' knowledge it is currently the only method for solving this modified version of the GPR model.
\end{abstract}

Keywords: Godunov-Peshkov-Romenski, GPR, Non-Newtonian, Plasticity, Operator Splitting

\section{Introduction}

\subsection{Background}

The Godunov-Pehskov-Romenski (GPR) model of continuum mechanics (see Peshkov and Romenski 21]) has been purported to represent an alternative formulation to describe both fluids and solids within the same hyperbolic system of differential equations. From a practical perspective, the potential ramifications of this include: the simplification of software made for the simulation of phenomena involving different states of matter (as commented on in Jackson [14); and the use of the vast array of effective numerical solvers designed for first-order hyperbolic systems. From a theoretical perspective, an advantage of the GPR model is that it cannot produce waves of infinite speed, unlike the parabolic Navier-Stokes equations. Additionally, the first-principles derivation of the mechanism by which viscous effects appear under the GPR model has been commented to be more appropriate than the more phenomenological viscous law appearing in the traditional Navier-Stokes formulation (see Peshkov et al. [22]).

Thus far, the GPR model has been solved for a wide array of different fluids (inviscid and viscous Newtonian) and solids (elastic and elastoplastic) (see Dumbser et al. 6, Boscheri et al. [4, Peshkov et al. 20, 22]). It has also been extended to incorporate the effects of electrodynamics (see Dumbser et al. [7]) and general relativity (see Peshkov et al. 23]). To the best of our knowledge, it is yet to be formally extended to include non-Newtonian power-law fluids, however. A method for doing so is presented in this paper. Building upon

\footnotetext{
${ }^{*}$ Corresponding author

** Principal corresponding author

Email address: hj305@cam.ac.uk (Haran Jackson)
} 
the work of Jackson [14] (in which a numerical scheme based upon a split solver was presented for Newtonian fluids and elastic solids under the GPR model) a numerical scheme is then presented in Section 3 for solving this new model. This scheme is adapted to work also for elastoplastic power-law materials. The scheme is validated against several $1 \mathrm{D}$ and 2D tests in Section 4, with discussion presented in Section 5 .

\subsection{The GPR Model}

The GPR model, first introduced in Peshkov and Romenski [21] - and expanded upon by Dumbser et al. 6] and Boscheri et al. [4] - takes the following form:

$$
\begin{aligned}
\frac{\partial \rho}{\partial t}+\frac{\partial\left(\rho v_{k}\right)}{\partial x_{k}} & =0 \\
\frac{\partial\left(\rho v_{i}\right)}{\partial t}+\frac{\partial\left(\rho v_{i} v_{k}+p \delta_{i k}-\sigma_{i k}\right)}{\partial x_{k}} & =0 \\
\frac{\partial A_{i j}}{\partial t}+\frac{\partial\left(A_{i k} v_{k}\right)}{\partial x_{j}}+v_{k}\left(\frac{\partial A_{i j}}{\partial x_{k}}-\frac{\partial A_{i k}}{\partial x_{j}}\right) & =-\frac{\psi_{i j}}{\theta_{1}} \\
\frac{\partial\left(\rho J_{i}\right)}{\partial t}+\frac{\partial\left(\rho J_{i} v_{k}+T \delta_{i k}\right)}{\partial x_{k}} & =-\frac{\rho H_{i}}{\theta_{2}} \\
\frac{\partial(\rho E)}{\partial t}+\frac{\partial\left(\rho E v_{k}+\left(p \delta_{i k}-\sigma_{i k}\right) v_{i}+q_{k}\right)}{\partial x_{k}} & =0
\end{aligned}
$$

where $\theta_{1}$ and $\theta_{2}$ are positive scalar functions, and $\psi=\frac{\partial E}{\partial A}$ and $\boldsymbol{H}=\frac{\partial E}{\partial \boldsymbol{J}}$. The following definitions are given:

$$
\begin{aligned}
p & =\left.\rho^{2} \frac{\partial E}{\partial \rho}\right|_{s, A} \\
\sigma & =-\left.\rho A^{T} \frac{\partial E}{\partial A}\right|_{\rho, s} \\
T & =\left.\frac{\partial E}{\partial s}\right|_{\rho, A} \\
\boldsymbol{q} & =T \frac{\partial E}{\partial \boldsymbol{J}}
\end{aligned}
$$

To close the system, the EOS must be specified, from which the above quantities and the sources can be derived. $E$ is the sum of the contributions of the energies at the molecular scale (microscale), the material element ${ }^{1}$ scale (mesoscale), and the flow scale (macroscale):

$$
E=E_{1}(\rho, s)+E_{2}(\rho, s, A, \boldsymbol{J})+E_{3}(\boldsymbol{v})
$$

Here, as in previous studies, such as Dumbser et al. [6] and Boscheri et al. [4, $E_{1}$ is taken to be either the ideal gas EOS, a shock Mie-Gruneisen EOS, or the EOS of nonlinear hyperelasticity (see Barton et al. [1]). $E_{2}$ has the following quadratic form:

\footnotetext{
${ }^{1}$ The concept of a material element corresponds to that of a fluid parcel from fluid dynamics, applied to both fluids and solids.
} 


$$
E_{2}=\frac{c_{s}(\rho, s)^{2}}{4}\|\operatorname{dev}(G)\|_{F}^{2}+\frac{c_{t}(\rho, s)^{2}}{2}\|\boldsymbol{J}\|^{2}
$$

$c_{s}$ is the characteristic velocity of transverse perturbations. $c_{t}$ is related to the characteristic velocity of propagation of heat waves 2 ,

$$
c_{h}=\frac{c_{t}}{\rho} \sqrt{\frac{T}{c_{v}}}
$$

In previous studies, $c_{t}$ has been taken to be constant, as it will be in this study.

$G=A^{T} A$ is the Gramian matrix of the distortion tensor, and $\operatorname{dev}(G)$ is the deviator (trace-free part) of $G$ :

$$
\operatorname{dev}(G)=G-\frac{1}{3} \operatorname{tr}(G) I
$$

$E_{3}$ is the usual specific kinetic energy per unit mass:

$$
E_{3}=\frac{1}{2}\|\boldsymbol{v}\|^{2}
$$

The following forms are taken:

$$
\begin{gathered}
\theta_{1}=\frac{\tau_{1} c_{s}^{2}}{3|A|^{\frac{5}{3}}} \\
\theta_{2}=\tau_{2} c_{t}^{2} \frac{\rho T_{0}}{\rho_{0} T} \\
\tau_{1}= \begin{cases}\frac{6 \mu}{\rho_{0} c_{s}^{2}} & \text { viscous fluids } \\
\tau_{0}\left(\frac{\sigma_{0}}{\|\operatorname{dev}(\sigma)\|_{F}}\right)^{n} & \text { elastoplastic solids } \\
\tau_{2}=\frac{\rho_{0} \kappa}{T_{0} c_{t}^{2}} & \end{cases}
\end{gathered}
$$

The justification of these choices is that classical Navier-Stokes-Fourier theory is recovered in the stiff limit $\tau_{1}, \tau_{2} \rightarrow 0$ (see Dumbser et al. [6]). The power law for elastoplastic solids is based on material from Barton et al. [2].

Finally, it is straightforward to verify that as a consequence of $(2 \mathrm{a}), 2 \mathrm{~b}, \mathrm{2c}, \mathrm{2d}$, we have the following relations:

$$
\begin{aligned}
\sigma & =-\rho c_{s}^{2} G \operatorname{dev}(G) \\
\boldsymbol{q} & =c_{t}^{2} T \boldsymbol{J} \\
-\frac{\psi}{\theta_{1}\left(\tau_{1}\right)} & =-\frac{3}{\tau_{1}}|A|^{\frac{5}{3}} A \operatorname{dev}(G) \\
-\frac{\rho \boldsymbol{H}}{\theta_{2}\left(\tau_{2}\right)} & =-\frac{T \rho_{0}}{T_{0} \tau_{2}} \boldsymbol{J}
\end{aligned}
$$

\footnotetext{
${ }^{2}$ Note that Dumbser et al. [6] denotes this variable by $\alpha$, which is avoided here due to a clash with a parameter of one of the equations of state used.
} 


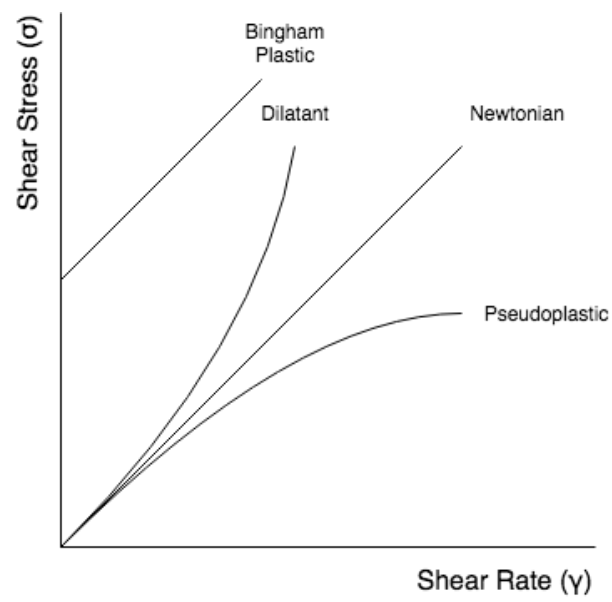

Figure 1: The stress-strain relationships for different kinds of fluids

The following constraint also holds (see Peshkov and Romenski [21]):

$$
\operatorname{det}(A)=\frac{\rho}{\rho_{0}}
$$

The GPR model and Godunov and Romenski's 1970s model of elastoplastic deformation in fact rely upon the same equations. The realization of Peshkov and Romenski was that these are the equations of motion for an arbitrary continuum - not just a solid - and so the model can be applied to fluids too. Unlike in previous continuum models, material elements have not only finite size, but also internal structure, encoded in the distortion tensor.

The strain dissipation time $\tau_{1}$ of the GPR model is a continuous analogue of Frenkel's "particle settled life time" (detailed in Frenkel [12]); the characteristic time taken for a particle to move by a distance of the same order of magnitude as the particle's size. Thus, $\tau_{1}$ characterizes the time taken for a material element to rearrange with its neighbors. $\tau_{1}=\infty$ for solids and $\tau_{1}=0$ for inviscid fluids. It is in this way that the GPR model seeks to describe all three major phases of matter, as long as a continuum description is appropriate for the material at hand.

The evolution equation for $\boldsymbol{J}$ and its contribution to the energy of the system are derived from Romenski's model of hyperbolic heat transfer, originally proposed in Malyshev and Romenskii [17], Romenski [26], and implemented in Romenski et al. [25, 24]. In this model, $\boldsymbol{J}$ is effectively defined as the variable conjugate to the entropy flux, in the sense that the latter is the derivative of the specific internal energy with respect to $\boldsymbol{J}$. Romenski remarks that it is more convenient to evolve $\boldsymbol{J}$ and $E$ than the heat flux or the entropy flux, and thus the equations take the form given here. $\tau_{2}$ characterizes the speed of relaxation of the thermal impulse due to heat exchange between material elements.

\section{Power-Law Fluids}

The stress-strain relationships for various kinds of fluids are shown in Figure 1 on page 4 Dilatants and pseudoplastics may be modelled using the following power law, with $n>1$ and $0<n<1$, respectively:

$$
\begin{aligned}
& \boldsymbol{\sigma}=K|\dot{\gamma}|^{n-1} \dot{\gamma} \\
& \dot{\gamma}=\nabla \boldsymbol{v}+\nabla \boldsymbol{v}^{T}-\frac{2 \operatorname{tr}(\nabla \boldsymbol{v})}{3} I
\end{aligned}
$$


$K>0$ is known as the consistency, and $K|\dot{\gamma}|^{n-1}$ is the apparent viscosity. The norm is taken to be:

$$
|X|=\sqrt{\frac{1}{2} X_{i j} X_{i j}}=\frac{\|X\|_{F}}{\sqrt{2}}
$$

In Dumbser et al. [6] it was noted that when expressing the state variables as an asymptotic expansion in the relaxation parameter $\tau_{1}$, to first order we have:

$$
\boldsymbol{\sigma}=\frac{1}{6} \tau_{1} \rho_{0} c_{s}^{2}\left(\nabla \boldsymbol{v}+\nabla \boldsymbol{v}^{T}-\frac{2}{3} \operatorname{tr}(\nabla \boldsymbol{v}) \boldsymbol{I}\right)
$$

Thus, for a power law fluid, we require that:

$$
\frac{1}{6} \tau_{1} \rho_{0} c_{s}^{2}=K|\dot{\gamma}|^{n-1}
$$

Taking moduli of both sides of $(15)$, we also have:

$$
|\boldsymbol{\sigma}|=\frac{1}{6} \tau_{1} \rho_{0} c_{s}^{2}|\dot{\gamma}|
$$

Combining these two relationships, we obtain:

$$
\tau_{1}=\frac{6 K^{\frac{1}{n}}}{\rho_{0} c_{s}^{2}}\left|\frac{1}{\boldsymbol{\sigma}}\right|^{\frac{1-n}{n}}:=\tau_{0}\left|\frac{1}{\boldsymbol{\sigma}}\right|^{\frac{1-n}{n}}
$$

\section{Numerical Schemes}

Note that 1a, 1b, 1c), 1d, 1e can be written in the following form:

$$
\frac{\partial \boldsymbol{Q}}{\partial t}+\nabla \cdot \boldsymbol{F}(\boldsymbol{Q})+\boldsymbol{B}(\boldsymbol{Q}) \cdot \nabla \boldsymbol{Q}=\boldsymbol{S}(\boldsymbol{Q})
$$

As described in Toro [28, a viable way to solve inhomogeneous systems of PDEs is to employ an operator splitting. That is, the following subsystems are solved:

$$
\begin{aligned}
\frac{\partial \boldsymbol{Q}}{\partial t}+\nabla \cdot \boldsymbol{F}(\boldsymbol{Q})+\boldsymbol{B}(\boldsymbol{Q}) \cdot \nabla \boldsymbol{Q} & =\mathbf{0} \\
\frac{d \boldsymbol{Q}}{d t} & =\boldsymbol{S}(\boldsymbol{Q})
\end{aligned}
$$

The advantage of this approach is that specialized solvers can be employed to compute the results of the different subsystems. Let $H^{\delta t}, S^{\delta t}$ be the operators that take data $\boldsymbol{Q}(x, t)$ to $\boldsymbol{Q}(x, t+\delta t)$ under systems 20a and 20b respectively. A second-order scheme (in time) for solving the full set of PDEs over time step $[0, \Delta t]$ is obtained by calculating $\boldsymbol{Q}_{\boldsymbol{\Delta} \boldsymbol{t}}$ using a Strang splitting:

$$
Q_{\Delta t}=S^{\frac{\Delta t}{2}} H^{\Delta t} S^{\frac{\Delta t}{2}} Q_{0}
$$


In the scheme proposed here, the homogeneous subsystem will be solved using a WENO reconstruction of the data, followed by a finite volume update, and the temporal ODEs will be solved with appropriate ODE solvers. It should be noted that there are other choices of solvers for the homogeneous system that could have been made (e.g. see MUSCL, SLIC, and WAF, among others in Toro [28]). The WENO method was chosen due to the arbitrarily high-order spatial reconstructions it is able to produce.

Noting that $\frac{d \rho}{d t}=0$ over the ODE time step, the operator $S$ entails solving the following systems:

$$
\begin{aligned}
& \frac{d A}{d t}=\frac{-3}{\tau_{1}}|A|^{\frac{5}{3}} A \operatorname{dev}(G) \\
& \frac{d \boldsymbol{J}}{d t}=-\frac{1}{\tau_{2}} \frac{T \rho_{0}}{T_{0} \rho} \boldsymbol{J}
\end{aligned}
$$

These systems can be solved concurrently with a stiff ODE solver. The Jacobians of these two systems to be used in an ODE solver are given in the appendix of Jackson [14. However, these systems can also be solved separately, using the analytical results presented in 3.2 and 3.3 , under specific assumptions. The second-order Strang splitting is then:

$$
Q_{\Delta t}=D^{\frac{\Delta t}{2}} T^{\frac{\Delta t}{2}} H^{\Delta t} T^{\frac{\Delta t}{2}} D^{\frac{\Delta t}{2}} Q_{0}
$$

where $D^{\delta t}, T^{\delta t}$ are the operators solving the distortion and thermal impulse ODEs respectively, over time step $\delta t$. This allows us to bypass the relatively computationally costly process of solving these systems numerically.

\subsection{The Homogeneous System}

A WENO reconstruction of the cell-averaged data is performed at the start of the time step (as described in Dumbser et al. [10]). Focusing on a single cell $C_{i}$ at time $t_{n}$, we have $\boldsymbol{w}^{\boldsymbol{n}}(\boldsymbol{x})=\boldsymbol{w}_{p}{ }_{p} \Psi_{p}(\boldsymbol{\chi}(\boldsymbol{x}))$ in $C_{i}$ where $\Psi_{p}$ is a tensor product of basis functions in each of the spatial dimensions. The flux in $C$ is approximated by $\boldsymbol{F}(\boldsymbol{x}) \approx \boldsymbol{F}\left(\boldsymbol{w}_{p}\right) \Psi_{p}(\boldsymbol{\chi}(\boldsymbol{x}))$. $\boldsymbol{w}_{p}$ are stepped forwards half a time step using the update formula:

$$
\begin{aligned}
\frac{\boldsymbol{w}_{\boldsymbol{p}}^{n+\frac{1}{2}}-\boldsymbol{w}_{\boldsymbol{p}}^{\boldsymbol{n}}}{\Delta t / 2}= & -\boldsymbol{F}\left(\boldsymbol{w}_{\boldsymbol{k}}^{\boldsymbol{n}}\right) \cdot \nabla \Psi_{k}\left(\chi_{\boldsymbol{p}}\right) \\
& -\boldsymbol{B}\left(\boldsymbol{w}_{\boldsymbol{p}}^{\boldsymbol{n}}\right) \cdot\left(\boldsymbol{w}_{\boldsymbol{k}}^{n} \nabla \Psi_{k}\left(\chi_{\boldsymbol{p}}\right)\right)
\end{aligned}
$$

i.e.

$$
\boldsymbol{w}_{\boldsymbol{p}}^{n+\frac{1}{2}}=\boldsymbol{w}_{\boldsymbol{p}}^{\boldsymbol{n}}-\frac{\Delta t}{2 \Delta x}\left(\begin{array}{c}
\boldsymbol{F}\left(\boldsymbol{w}_{\boldsymbol{k}}^{n}\right) \cdot \nabla \Psi_{k}\left(\chi_{\boldsymbol{p}}\right) \\
+\boldsymbol{B}\left(\boldsymbol{w}_{\boldsymbol{p}}^{\boldsymbol{n}}\right) \cdot\left(\boldsymbol{w}_{\boldsymbol{k}}^{n} \nabla \Psi_{k}\left(\chi_{\boldsymbol{p}}\right)\right)
\end{array}\right)
$$

where $\chi_{\boldsymbol{p}}$ is the node corresponding to $\Psi_{p}$. This evolution to the middle of the time step is similar to that used in the second-order MUSCL and SLIC schemes (see Toro [28]) and, as with those schemes, it is integral to giving the method presented here its second-order accuracy.

Integrating 20a over $C$ gives:

$$
Q_{i}^{n+1}=Q_{i}^{n}-\Delta t_{n}\left(P_{i}^{n+\frac{1}{2}}+D_{i}^{n+\frac{1}{2}}\right)
$$


where

$$
\begin{aligned}
\boldsymbol{Q}_{\boldsymbol{i}}^{\boldsymbol{n}} & =\frac{1}{V} \int_{C} \boldsymbol{Q}\left(\boldsymbol{x}, t_{n}\right) d \boldsymbol{x} \\
\boldsymbol{P}_{\boldsymbol{i}}^{\boldsymbol{n}+\frac{1}{2}} & =\frac{1}{V} \int_{C} \boldsymbol{B}\left(\boldsymbol{Q}\left(\boldsymbol{x}, t_{n+\frac{1}{2}}\right)\right) \cdot \nabla \boldsymbol{Q}\left(\boldsymbol{x}, t_{n+\frac{1}{2}}\right) d \boldsymbol{x} \\
\boldsymbol{D}_{\boldsymbol{i}}^{n+\frac{1}{2}} & =\frac{1}{V} \oint_{\partial C} \mathcal{D}\left(\boldsymbol{Q}^{-}\left(\boldsymbol{s}, t_{n+\frac{1}{2}}\right), \boldsymbol{Q}^{+}\left(\boldsymbol{s}, t_{n+\frac{1}{2}}\right)\right) d \boldsymbol{s}
\end{aligned}
$$

where $V$ is the volume of $C$ and $Q^{-}, Q^{+}$are the interior and exterior extrapolated states at the boundary of $C$, respectively.

Note that 20a can be rewritten as:

$$
\frac{\partial \boldsymbol{Q}}{\partial t}+\boldsymbol{M}(\boldsymbol{Q}) \cdot \nabla \boldsymbol{Q}=\mathbf{0}
$$

where $\boldsymbol{M}=\frac{\partial \boldsymbol{F}}{\partial \boldsymbol{Q}}+\boldsymbol{B}$. Let $\boldsymbol{n}$ be the normal to the boundary at point $\boldsymbol{s} \in \partial C$. For the GPR model, $\hat{M}=\boldsymbol{M}(\boldsymbol{Q}(\boldsymbol{s})) \cdot \boldsymbol{n}$ is a diagonalizable matrix with decomposition $\hat{M}=\hat{R} \hat{\Lambda} \hat{R}^{-1}$ where the columns of $\hat{R}$ are the right eigenvectors and $\hat{\Lambda}$ is the diagonal matrix of eigenvalues. Define also $\hat{\boldsymbol{F}}=\boldsymbol{F} \cdot \boldsymbol{n}$ and $\hat{B}=\boldsymbol{B} \cdot \boldsymbol{n}$. Using these definitions, the interface terms arising in the FV formula have the following form:

$$
\begin{aligned}
\mathcal{D}\left(\boldsymbol{Q}^{-}, \boldsymbol{Q}^{+}\right) & =\frac{1}{2}\left(\hat{\boldsymbol{F}}\left(\boldsymbol{Q}^{+}\right)+\hat{\boldsymbol{F}}\left(\boldsymbol{Q}^{-}\right)\right) \\
& +\frac{1}{2}\left(+\tilde{B}\left(\boldsymbol{Q}^{+}-\boldsymbol{Q}^{-}\right)+\tilde{M}\left(\boldsymbol{Q}^{+}-\boldsymbol{Q}^{-}\right)\right)
\end{aligned}
$$

$\tilde{M}$ is chosen to either correspond to a Rusanov/Lax-Friedrichs flux (see Toro [28]):

$$
\tilde{M}=\max \left(\max \left|\hat{\Lambda}\left(\boldsymbol{Q}^{+}\right)\right|, \max \left|\hat{\Lambda}\left(\boldsymbol{Q}^{-}\right)\right|\right)
$$

or a Roe flux (see Dumbser and Toro [9]):

$$
\hat{M}=\left|\int_{0}^{1} M\left(\boldsymbol{q}^{-}+z\left(\boldsymbol{q}^{+}-\boldsymbol{q}^{-}\right)\right) d z\right|
$$

or a simplified Osher-Solomon flux (see Dumbser and Toro [9, 8]):

$$
\tilde{M}=\int_{0}^{1}\left|\hat{M}\left(\boldsymbol{Q}^{-}+z\left(\boldsymbol{Q}^{+}-\boldsymbol{Q}^{-}\right)\right)\right| d z
$$

where

$$
|\hat{M}|=\hat{R}|\hat{\Lambda}| \hat{R}^{-1}
$$

$\tilde{B}$ takes the following form: 


$$
\tilde{B}=\int_{0}^{1} \hat{B}\left(\boldsymbol{Q}^{-}+z\left(\boldsymbol{Q}^{+}-\boldsymbol{Q}^{-}\right)\right) d z
$$

$\boldsymbol{P}_{i}^{n+\frac{1}{2}}, \boldsymbol{D}_{i}^{n+\frac{1}{2}}$ are calculated using an $N+1$-point Gauss-Legendre quadrature, replacing $\boldsymbol{Q}\left(\boldsymbol{x}, t_{n+\frac{1}{2}}\right)$ with $w^{n+\frac{1}{2}}(x)$.

\subsection{The Thermal Impulse ODEs}

The following analytical solution to the thermal impulse ODEs was first presented in Jackson [14. It is included here for completeness.

Taking the EOS for the GPR model (3) and denoting by $E_{2}^{(A)}, E_{2}^{(J)}$ the components of $E_{2}$ depending on $A$ and $\boldsymbol{J}$ respectively, we have:

$$
\begin{aligned}
T & =\frac{E_{1}}{c_{v}} \\
& =\frac{E-E_{2}^{(A)}(\rho, s, A)-E_{3}(\boldsymbol{v})}{c_{v}}-\frac{1}{c_{v}} E_{2}^{(J)}(\boldsymbol{J}) \\
& =c_{1}-c_{2}\|\boldsymbol{J}\|^{2}
\end{aligned}
$$

where:

$$
\begin{aligned}
c_{1} & =\frac{E-E_{2}^{(A)}(A)-E_{3}(\boldsymbol{v})}{c_{v}} \\
c_{2} & =\frac{c_{t}^{2}}{2 c_{v}}
\end{aligned}
$$

Over the time period of the ODE $22 \mathrm{~b}, c_{1}, c_{2}>0$ are constant. We have:

$$
\frac{d J_{i}}{d t}=-\left(\frac{1}{\tau_{2}} \frac{\rho_{0}}{T_{0} \rho}\right) J_{i}\left(c_{1}-c_{2}\|\boldsymbol{J}\|^{2}\right)
$$

Therefore:

$$
\frac{d}{d t}\left(J_{i}^{2}\right)=J_{i}^{2}\left(-a+b\left(J_{1}^{2}+J_{2}^{2}+J_{3}^{2}\right)\right)
$$

where

$$
\begin{aligned}
a & =\frac{2 \rho_{0}}{\tau_{2} T_{0} \rho c_{v}}\left(E-E_{2}^{(A)}(A)-E_{3}(\boldsymbol{v})\right) \\
b & =\frac{\rho_{0} c_{t}^{2}}{\tau_{2} T_{0} \rho c_{v}}
\end{aligned}
$$

Note that this is a generalized Lotka-Volterra system in $\left\{J_{1}^{2}, J_{2}^{2}, J_{3}^{2}\right\}$. It has the following analytical solution:

$$
\boldsymbol{J}(t)=\boldsymbol{J}(0) \sqrt{\frac{1}{e^{a t}-\frac{b}{a}\left(e^{a t}-1\right)\|\boldsymbol{J}(0)\|^{2}}}
$$




\subsection{The Distortion ODEs}

The following analytical solution to the distortion ODEs for Newtonian fluids was first presented in Jackson 14. It is included here, as the solutions for non-Newtonian fluids and elastoplastic solids depend on the Newtonian solution.

\subsubsection{Newontian Fluids}

Let $k_{0}=\frac{3}{\tau_{1}}\left(\frac{\rho}{\rho_{0}}\right)^{\frac{5}{3}}>0$ and let $A$ have singular value decomposition $U \Sigma V^{T}$. Then:

$$
\begin{gathered}
G=\left(U \Sigma V^{T}\right)^{T} U \Sigma V^{T}=V \Sigma^{2} V^{T} \\
\operatorname{tr}(G)=\operatorname{tr}\left(V \Sigma^{2} V^{T}\right)=\operatorname{tr}\left(\Sigma^{2} V^{T} V\right)=\operatorname{tr}\left(\Sigma^{2}\right)
\end{gathered}
$$

Therefore:

$$
\begin{aligned}
\frac{d A}{d t} & =-k_{0} U \Sigma V^{T}\left(V \Sigma^{2} V^{T}-\frac{\operatorname{tr}\left(\Sigma^{2}\right)}{3} I\right) \\
& =-k_{0} U \Sigma\left(\Sigma^{2}-\frac{\operatorname{tr}\left(\Sigma^{2}\right)}{3}\right) V^{T} \\
& =-k_{0} U \Sigma \operatorname{dev}\left(\Sigma^{2}\right) V^{T}
\end{aligned}
$$

It is a common result (see Giles [13]) that:

$$
d \Sigma=U^{T} d A V
$$

and thus:

$$
\frac{d \Sigma}{d t}=-k_{0} \Sigma \operatorname{dev}\left(\Sigma^{2}\right)
$$

Using a fast $3 \times 3 \mathrm{SVD}$ algorithm (such as in McAdams et al. [18), $U, V, \Sigma$ can be obtained, after which the following procedure is applied to $\Sigma$, giving $A(t)=U \Sigma(t) V^{T}$.

Denote the singular values of $A$ by $a_{1}, a_{2}, a_{3}$. Then:

$$
\Sigma \operatorname{dev}\left(\Sigma^{2}\right)=\left(\begin{array}{ccc}
a_{1}\left(a_{1}^{2}-\alpha\right) & 0 & 0 \\
0 & a_{1}\left(a_{1}^{2}-\alpha\right) & 0 \\
0 & 0 & a_{1}\left(a_{1}^{2}-\alpha\right)
\end{array}\right)
$$

where

$$
\alpha=\frac{a_{1}^{2}+a_{2}^{2}+a_{3}^{2}}{3}
$$

Letting $x_{i}=\frac{a_{i}^{2}}{\operatorname{det}(A)^{\frac{2}{3}}}=\frac{a_{i}^{2}}{\left(\frac{\rho}{\rho_{0}}\right)^{\frac{2}{3}}}$ we have: 


$$
\frac{d x_{i}}{d \tau}=-3 x_{i}\left(x_{i}-\bar{x}\right)
$$

where $\tau=\frac{2}{\tau_{1}}\left(\frac{\rho}{\rho_{0}}\right)^{\frac{7}{3}} t$ and $\bar{x}$ is the arithmetic mean of $x_{1}, x_{2}, x_{3}$. This ODE system travels along the surface $\Psi=\left\{x_{1}, x_{2}, x_{3}>0, x_{1} x_{2} x_{3}=1\right\}$ to the point $x_{1}, x_{2}, x_{3}=1$. This surface is symmetrical in the planes $x_{1}=x_{2}, x_{1}=x_{3}, x_{2}=x_{3}$. As such, given that the system is autonomous, the paths of evolution of the $x_{i}$ cannot cross the intersections of these planes with $\Psi$. Thus, any non-strict inequality of the form $x_{i} \geq x_{j} \geq x_{k}$ is maintained for the whole history of the system. By considering (48) it is clear that in this case $x_{i}$ is monotone decreasing, $x_{k}$ is monotone increasing, and the time derivative of $x_{j}$ may switch sign.

We now explore cases when even the reduced ODE system (48) need not be solved numerically. Define the following variables:

$$
\begin{aligned}
m & =\frac{x_{1}+x_{2}+x_{3}}{3} \\
u & =\frac{\left(x_{1}-x_{2}\right)^{2}+\left(x_{2}-x_{3}\right)^{2}+\left(x_{3}-x_{1}\right)^{2}}{3}
\end{aligned}
$$

It is a standard result that $m \geq \sqrt[3]{x_{1} x_{2} x_{3}}$. Thus, $m \geq 1$. Note that $u$ is proportional to the internal energy contribution from the distortion. From $(48)$ we have:

$$
\begin{aligned}
\frac{d u}{d \tau} & =-18\left(1-m\left(m^{2}-\frac{5}{6} u\right)\right) \\
\frac{d m}{d \tau} & =-u
\end{aligned}
$$

Combining these equations, we have:

$$
\frac{d^{2} m}{d \tau^{2}}=-\frac{d u}{d \tau}=18\left(1-m\left(m^{2}-\frac{5}{6} u\right)\right)
$$

Therefore:

$$
\left\{\begin{array}{c}
\frac{d^{2} m}{d \tau^{2}}+15 m \frac{d m}{d \tau}+18\left(m^{3}-1\right)=0 \\
m(0)=m_{0} \\
m^{\prime}(0)=-u_{0}
\end{array}\right.
$$

We make the following assumption, noting that it is true in all physical situations tested in this study:

$$
m(t)=1+\eta(t), \quad \eta \ll 1 \forall t \geq 0
$$

Thus, we have the linearized ODE:

$$
\left\{\begin{array}{c}
\frac{d^{2} \eta}{d \tau^{2}}+15 \frac{d \eta}{d \tau}+54 \eta=0 \\
\eta(0)=m_{0}-1 \\
\eta^{\prime}(0)=-u_{0}
\end{array}\right.
$$


This is a Sturm-Liouville equation with solution:

$$
\eta(\tau)=\frac{e^{-9 \tau}}{3}\left(a e^{3 \tau}-b\right)
$$

where

$$
\begin{aligned}
& a=9 m_{0}-u_{0}-9 \\
& b=6 m_{0}-u_{0}-6
\end{aligned}
$$

Thus, we also have:

$$
u(\tau)=e^{-9 \tau}\left(2 a e^{3 \tau}-3 b\right)
$$

Denote the following:

$$
\begin{aligned}
m_{\Delta t} & =1+\eta\left(\frac{2}{\tau_{1}}\left(\frac{\rho}{\rho_{0}}\right)^{\frac{7}{3}} \Delta t\right) \\
u_{\Delta t} & =u\left(\frac{2}{\tau_{1}}\left(\frac{\rho}{\rho_{0}}\right)^{\frac{7}{3}} \Delta t\right)
\end{aligned}
$$

Once these have been found, we have:

$$
\begin{aligned}
\frac{x_{i}+x_{j}+x_{k}}{3} & =m_{\Delta t} \\
\frac{\left(x_{i}-x_{j}\right)^{2}+\left(x_{j}-x_{k}\right)^{2}+\left(x_{k}-x_{i}\right)^{2}}{3} & =u_{\Delta t} \\
3 & =1
\end{aligned}
$$

This gives:

$$
\begin{aligned}
& x_{i}=\frac{\Xi}{6}+\frac{u_{\Delta t}}{\Xi}+m_{\Delta t} \\
& x_{j}=\frac{1}{2}\left(\sqrt{\frac{x_{i}\left(3 m_{\Delta t}-x_{i}\right)^{2}-4}{x_{i}}}+3 m_{\Delta t}-x_{i}\right) \\
& x_{k}=\frac{1}{x_{i} x_{j}}
\end{aligned}
$$

where

$$
\begin{aligned}
& \Xi=\sqrt[3]{6\left(\sqrt{81 \Delta^{2}-6 u_{\Delta t}^{3}}+9 \Delta\right)} \\
& \Delta=-2 m_{\Delta t}^{3}+m_{\Delta t} u_{\Delta t}+2
\end{aligned}
$$


Note that taking the real parts of the above expression for $x_{i}$ gives:

$$
\begin{aligned}
x_{i} & =\frac{\sqrt{6 u_{\Delta t}}}{3} \cos \left(\frac{\theta}{3}\right)+m_{\Delta t} \\
\theta & =\tan ^{-1}\left(\frac{\sqrt{6 u_{\Delta t}^{3}-81 \Delta^{2}}}{9 \Delta}\right)
\end{aligned}
$$

At this point it is not clear which values of $\left\{x_{i}, x_{j}, x_{k}\right\}$ are taken by $x_{1}, x_{2}, x_{3}$. However, this can be inferred from the fact that any relation $x_{i} \geq x_{j} \geq x_{k}$ is maintained over the lifetime of the system. Thus, the stiff ODE solver has been obviated by a few arithmetic operations.

\subsubsection{Power Law Fluids}

Take the singular value decomposition $A=U \Sigma V^{T}$. Note that:

$$
\sigma=-\rho c_{s}^{2} A^{T} A \operatorname{dev}\left(A^{T} A\right)=-\rho c_{s}^{2} V \Sigma^{2} \operatorname{dev}\left(\Sigma^{2}\right) V^{T}
$$

Thus:

$$
\|\sigma\|_{F}^{k}=\rho^{k} c_{s}^{2 k}\left\|\Sigma^{2} \operatorname{dev}\left(\Sigma^{2}\right)\right\|_{F}^{k}
$$

Thus, according to 18 , and letting $k=\frac{1-n}{n}$, we have:

$$
\frac{d \Sigma}{d t}=-\frac{3}{\tau_{0}}\left(\frac{\rho}{\rho_{0}}\right)^{\frac{5}{3}} \frac{\rho^{k} c_{s}^{2 k}}{2^{\frac{k}{2}}}\left\|\Sigma^{2} \operatorname{dev}\left(\Sigma^{2}\right)\right\|_{F}^{k} \Sigma \operatorname{dev}\left(\Sigma^{2}\right)
$$

Letting $x_{i}=\frac{a_{i}^{2}}{\operatorname{det}(A)^{\frac{2}{3}}}=\frac{a_{i}^{2}}{\left(\frac{\rho}{\rho_{0}}\right)^{\frac{2}{3}}}$ then $\Sigma^{2}=\operatorname{det}(A)^{\frac{2}{3}} X$ where $X=\operatorname{diag}\left(x_{1}, x_{2}, x_{3}\right)$. Thus, we have:

$$
\frac{d x_{i}}{d \tilde{t}}=-3\|X \operatorname{dev}(X)\|_{F}^{k} x_{i}\left(x_{i}-\bar{x}\right)
$$

where:

$$
\tilde{t}=\frac{2}{\tau_{0}}\left(\frac{\rho}{\rho_{0}}\right)^{\frac{4 k+7}{3}}\left(\frac{\rho c_{s}^{2}}{\sqrt{2}}\right)^{k} t
$$

Note that:

$$
\begin{aligned}
9\|X \operatorname{dev}(X)\|_{F}^{2} & =4\left(x_{1}^{4}+x_{2}^{4}+x_{3}^{4}\right) \\
& -2\left(x_{1}^{2} x_{2}^{2}+x_{3}^{2} x_{2}^{2}+x_{1}^{2} x_{3}^{2}\right) \\
& +\sum_{i \neq j, j \neq k, k \neq i} x_{i}^{2} x_{j} x_{k}-4 \sum_{i \neq j} x_{i}^{3} x_{j}
\end{aligned}
$$

Defining $m, u$ as before, we have: 


$$
\|X \operatorname{dev}(X)\|_{F}^{2}=\frac{1}{2} u^{2}+4 m^{2} u-6 m^{4}+6 m
$$

This leads to the following coupled system of ODEs:

$$
\begin{aligned}
\frac{d u}{d \tilde{t}} & =-18 \frac{d \tau}{d \tilde{t}}\left(1-m\left(m^{2}-\frac{5}{6} u\right)\right) \\
\frac{d m}{d \tilde{t}} & =-\frac{d \tau}{d \tilde{t}} u
\end{aligned}
$$

where we have defined the variable $\tau$ by:

$$
\frac{d \tau}{d \tilde{t}}=\left(\frac{1}{2} u^{2}+4 m^{2} u-6 m^{4}+6 m\right)^{\frac{k}{2}}
$$

Using the approximation solution from before:

$$
\begin{aligned}
& m(\tau)=1+\frac{e^{-9 \tau}}{3}\left(a e^{3 \tau}-b\right) \\
& u(\tau)=e^{-9 \tau}\left(2 a e^{3 \tau}-3 b\right)
\end{aligned}
$$

It is straightforward to verify that:

$$
\begin{gathered}
\frac{d \tau}{d \tilde{t}}=\frac{1}{54^{\frac{k}{2}}}\left(\begin{array}{c}
108 a e^{-6 \tau}-324 b e^{-9 \tau} \\
+180 a^{2} e^{-12 \tau}-612 a b e^{-15 \tau} \\
+459 b^{2} e^{-18 \tau}-24 a^{2} b e^{-21 \tau} \\
+\left(48 a b^{2}-4 a^{4}\right) e^{-24 \tau} \\
+\left(16 a^{3} b-24 b^{3}\right) e^{-27 \tau} \\
-24 a^{2} b^{2} e^{-30 \tau}+16 a b^{3} e^{-33 \tau} \\
-4 b^{4} e^{-36 \tau}
\end{array}\right) \\
\equiv \frac{f(\tau)^{\frac{k}{2}}}{54^{\frac{k}{2}}}
\end{gathered}
$$

$f(\tau)$ is approximated by $g(\tau) \equiv c e^{-\frac{c}{\lambda} \tau}$, where:

$$
\begin{aligned}
c & =108 a-324 b+180 a^{2}-612 a b+459 b^{2} \\
& -24\left(a^{2} b-2 a b^{2}+b^{3}\right)-4(a-b)^{4} \\
\lambda & =18 a-36 b+15 a^{2}-\frac{204 a b}{5}+\frac{51 b^{2}}{2} \\
- & \frac{8 a^{2} b}{7}+2 a b^{2}-\frac{8 b^{3}}{9}-\frac{a^{4}}{6}+\frac{16 a^{3} b}{27} \\
- & \frac{4 a^{2} b^{2}}{5}+\frac{16 a b^{3}}{33}-\frac{b^{4}}{9}
\end{aligned}
$$


Note that $f(0)=g(0)$ and $\int_{0}^{\infty}(f(\tau)-g(\tau)) d \tau=0$. Thus, we have:

$$
\frac{d \tau}{d \tilde{t}} \approx\left(\frac{c}{54}\right)^{\frac{k}{2}} e^{-\frac{k c}{2 \lambda} \tau}
$$

Therefore:

$$
\begin{aligned}
\tau & \approx \frac{2 \lambda}{k c} \log \left(\frac{k c}{2 \lambda}\left(\frac{c}{54}\right)^{\frac{k}{2}} \tilde{t}+1\right) \\
& =\frac{2 \lambda}{k c} \log \left(\frac{k c}{\tau_{0} \lambda}\left(\frac{\rho}{\rho_{0}}\right)^{\frac{4 k+7}{3}}\left(\frac{\sqrt{c} \rho c_{s}^{2}}{6 \sqrt{3}}\right)^{k} t+1\right)
\end{aligned}
$$

\subsubsection{Elastoplastic Solids}

For elastoplastic materials governed by the power law described in 9a:

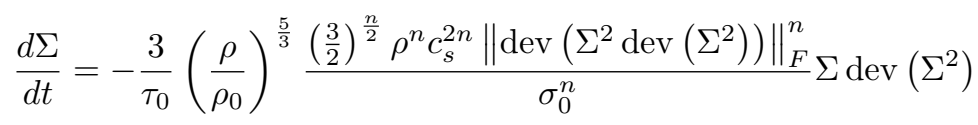

Thus, we have:

$$
\frac{d x_{i}}{d \tilde{t}}=-3\|\operatorname{dev}(X \operatorname{dev}(X))\|_{F}^{n} x_{i}\left(x_{i}-\bar{x}\right)
$$

where:

$$
\tilde{t}=\frac{2}{\tau_{0}}\left(\frac{\rho}{\rho_{0}}\right)^{\frac{4 n+7}{3}}\left(\sqrt{\frac{3}{2}} \frac{\rho c_{s}^{2}}{\sigma_{0}}\right)^{n} t
$$

Note that:

$$
\begin{aligned}
\frac{27}{2}\|\operatorname{dev}(X \operatorname{dev}(X))\|_{F}^{2} & =\frac{3}{2} \sum_{i \neq j, j \neq k, k \neq i} x_{i}^{2} x_{j} x_{k} \\
& -2 \sum_{i \neq j} x_{i}^{3} x_{j} \\
& -3\left(x_{1}^{2} x_{2}^{2}+x_{3}^{2} x_{2}^{2}+x_{1}^{2} x_{3}^{2}\right) \\
& +4\left(x_{1}^{4}+x_{2}^{4}+x_{3}^{4}\right)
\end{aligned}
$$

Thus we have:

$$
\|\operatorname{dev}(X \operatorname{dev}(X))\|_{F}^{2}=\frac{1}{6} u^{2}+4 m^{2} u-6 m^{4}+6 m
$$


This leads to the following coupled system of ODEs:

$$
\begin{aligned}
\frac{d u}{d \tilde{t}} & =-18 \frac{d \tau}{d \tilde{t}}\left(1-m\left(m^{2}-\frac{5}{6} u\right)\right) \\
\frac{d m}{d \tilde{t}} & =-\frac{d \tau}{d \tilde{t}} u
\end{aligned}
$$

where we have defined the variable $\tau$ by:

$$
\frac{d \tau}{d \tilde{t}}=\left(\frac{1}{6} u^{2}+4 m^{2} u-6 m^{4}+6 m\right)^{\frac{n}{2}}
$$

Then we have:

$$
\begin{aligned}
& \frac{d u}{d \tau}=-18\left(1-m\left(m^{2}-\frac{5}{6} u\right)\right) \\
& \frac{d m}{d \tau}=-u
\end{aligned}
$$

Using the approximate solution $72 \mathrm{a}, 72 \mathrm{~b}$ again, it is straightforward to verify that:

$$
\begin{gathered}
\frac{d \tau}{d \tilde{t}}=\frac{1}{54^{\frac{n}{2}}}\left(\begin{array}{c}
108 a e^{-6 \tau}-324 b e^{-9 \tau} \\
+108 a^{2} e^{-12 \tau}-396 a b e^{-15 \tau} \\
+297 b^{2} e^{-18 \tau}-24 a^{2} b e^{-21 \tau} \\
+\left(48 a b^{2}-4 a^{4}\right) e^{-24 \tau} \\
+\left(16 a^{3} b-24 b^{3}\right) e^{-27 \tau} \\
-24 a^{2} b^{2} e^{-30 \tau}+16 a b^{3} e^{-33 \tau} \\
-4 b^{4} e^{-36 \tau}
\end{array}\right) \\
\equiv \frac{f(\tau)^{\frac{n}{2}}}{54^{\frac{n}{2}}}
\end{gathered}
$$

$f(\tau)$ is approximated by $g(\tau) \equiv c e^{-\lambda \tau}$, where:

$$
\begin{aligned}
c & =108 a-324 b+108 a^{2}-396 a b+297 b^{2} \\
& -24\left(a^{2} b-2 a b^{2}+b^{3}\right)-4(a-b)^{4} \\
\lambda & =18 a-36 b+9 a^{2}-\frac{132 a b}{5}+\frac{33 b^{2}}{2} \\
& -\frac{8 a^{2} b}{7}+2 a b^{2}-\frac{8 b^{3}}{9}-\frac{a^{4}}{6} \\
& +\frac{16 a^{3} b}{27}-\frac{4 a^{2} b^{2}}{5}+\frac{16 a b^{3}}{33}-\frac{b^{4}}{9}
\end{aligned}
$$

Note that $f(0)=g(0)$ and $\int_{0}^{\infty}(f(\tau)-g(\tau)) d \tau=0$. Thus, we have: 


$$
\frac{d \tau}{d \tilde{t}} \approx\left(\frac{c}{54}\right)^{\frac{n}{2}} e^{-\frac{n c}{2 \lambda} \tau}
$$

Therefore:

$$
\begin{aligned}
\tau & \approx \frac{2 \lambda}{n c} \log \left(\frac{n c}{2 \lambda}\left(\frac{c}{54}\right)^{\frac{n}{2}} \tilde{t}+1\right) \\
& =\frac{2 \lambda}{n c} \log \left(\frac{n c}{\tau_{0} \lambda}\left(\frac{\rho}{\rho_{0}}\right)^{\frac{4 n+7}{3}}\left(\frac{\sqrt{c}}{6} \frac{\rho c_{s}^{2}}{\sigma_{0}}\right)^{n} t+1\right)
\end{aligned}
$$

Thus, the value of $A$ at time $\Delta t$ is found by substituting the following into $72 \mathrm{a}, 72 \mathrm{~b}$ :

$$
\tau=\frac{2 \lambda}{n c} \log \left(\frac{n c}{\tau_{0} \lambda}\left(\frac{\rho}{\rho_{0}}\right)^{\frac{4 n+7}{3}}\left(\frac{\sqrt{c}}{6} \frac{\rho c_{s}^{2}}{\sigma_{0}}\right)^{n} \Delta t+1\right)
$$

The results are in turn substituted into 62a, 60b, 60c.

\subsection{Distortion Correction in Fluids}

Owing to the linearization step in (54), the method presented will perform poorly if the mean of the normalized singular values of the distortion tensor, $m$, deviates significantly from 1 . To avert this, the following resetting procedure was applied globally for fluid flow problems when $m>1.03$ :

$$
\begin{aligned}
& E \mapsto E-\frac{c_{S}^{2}}{4}\|\operatorname{dev}(G)\|_{F}^{2} \\
& A \mapsto\left(\frac{\rho}{\rho_{0}}\right)^{1 / 3} I
\end{aligned}
$$

This is justified by the fact that the distortion tensor is not a macroscopically-measurable quantity. This transformation leaves the density, pressure, and velocity of the fluid unchanged, and was found to improve the stability of the numerical scheme, while at the same time producing correct results, as demonstrated in the following section.

\section{Numerical Results}

In this section, a variety of test problems are solved, with a dual purpose. Firstly, we demonstrate the ability of the modified GPR formulation presented in Section 2 to model power-law fluids. Secondly, we demonstrate the efficacy of the numerical schemes presented in Section 3 in solving this system, and the existing power-law plasticity formulation of the GPR model. 


\subsection{Strain Relaxation Test}

The aim of this test is to gauge the accuracy of the approximate analytic solver for the distortion equations.

Take initial data used by Barton:

$$
A=\left(\begin{array}{ccc}
1 & 0 & 0 \\
-0.01 & 0.95 & 0.02 \\
-0.015 & 0 & 0.9
\end{array}\right)^{-1}
$$

The following parameter values were used: $\rho_{0}=1, c_{s}=0.219, n=4, \sigma_{0}=9 \times 10^{-4}, \tau_{0}=0.1$.

The evolution of the components of the distortion tensor, according to both the approximate analytical solver and a stiff numerical ODE solver, are given in Figure 2 on page 18 . Figure 3 on page 18 , and Figure 4 on page 19. As can be seen, the approximate analytic solver compares well with the exact solution for the distortion tensor $A$, and thus also the stress tensor and the energy.

\subsection{Poiseuille Flow}

The aim of this test is to gauge both the performance of the modified formulation of the GPR model in simulating power-law fluids, and the accuracy of the new numerical scheme we have presented to solve it. The problem of poiseuille flow has been chosen due to the availability of an analytical solution against which to compare.

This test consists of a fluid traveling down a channel of constant width $L$, with a constant pressure gradient $\Delta p$ along the length of the channel. No-slip boundary conditions are imposed on the channel walls. For a non-Newtonian fluid obeying a power law, the steady-state velocity profile across the channel is given by Ferras et al. [11]:

$$
\begin{aligned}
& v=\frac{\rho}{k}\left(\frac{\Delta p}{K}\right)^{1 / n}\left(\left(\frac{L}{2}\right)^{k}-\left(x-\frac{L}{2}\right)^{k}\right) \\
& k=\frac{n+1}{n}
\end{aligned}
$$

where $x \in[0, L]$.

In this case, $L=0.25, \Delta p=0.48, K=10^{-2}$. The fluid is initially at rest, with $\rho_{0}=1, A=I, p=100 / \gamma$. It follows an ideal gas EOS with $\gamma=1.4, c_{s}=1$. The pressure gradient is imposed by means of a body force, implemented as a constant source term to the momentum equation.

The final time was taken to be 20 , so that in each case the system had reached steady state. 100 cells were taken across the width of the channel. A third order WENO method was used, with a CFL number of 0.6.

Results for various values of $n$ are shown in Figure 5 on page 19 . The exact solutions are shown as dotted lines, with the numerical solutions in solid colors. Note that there is good agreement between the numerical solutions and exact solutions for all values of $n$.

\subsection{Lid-Driven Cavity}

This lid-driven cavity test has been chosen here as a famous multidimensional problem against which the power-law fluid framework we have presented can be benchmarked. See Sverdrup et al. [27] for detailed analysis of this problem, under power-law fluids and other non-Newtonian fluids. 

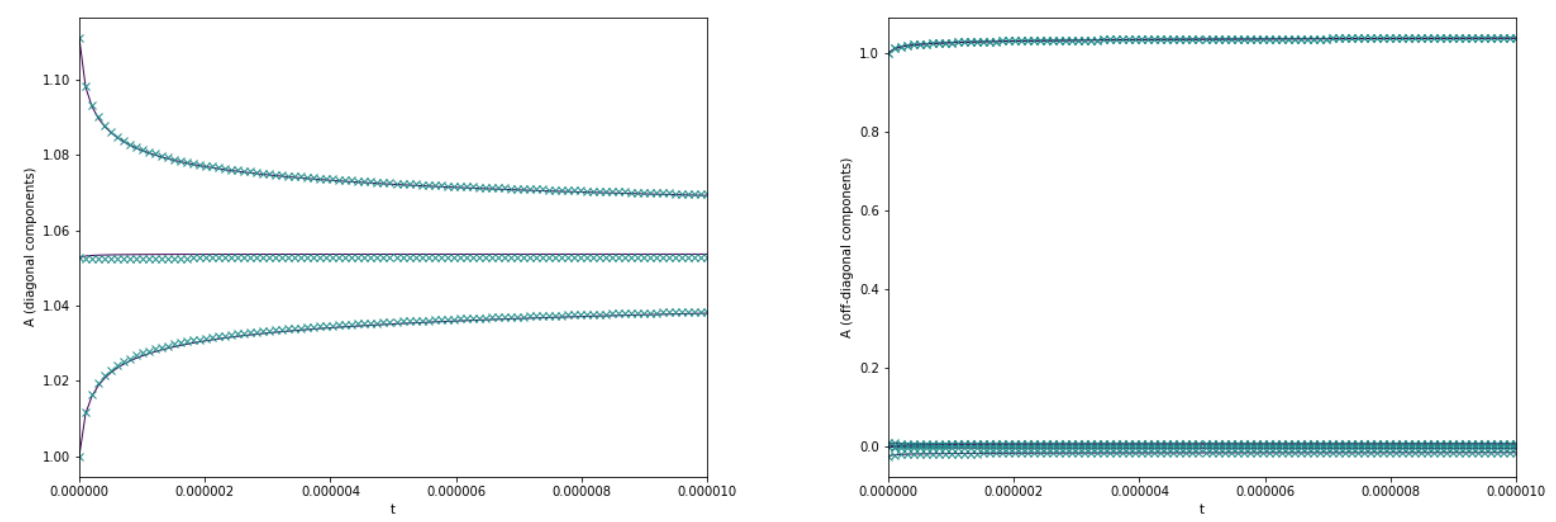

Figure 2: Distortion tensor components during the Strain Relaxation Test: approximate analytical solution (crosses) and numerical ODE solution (solid line)
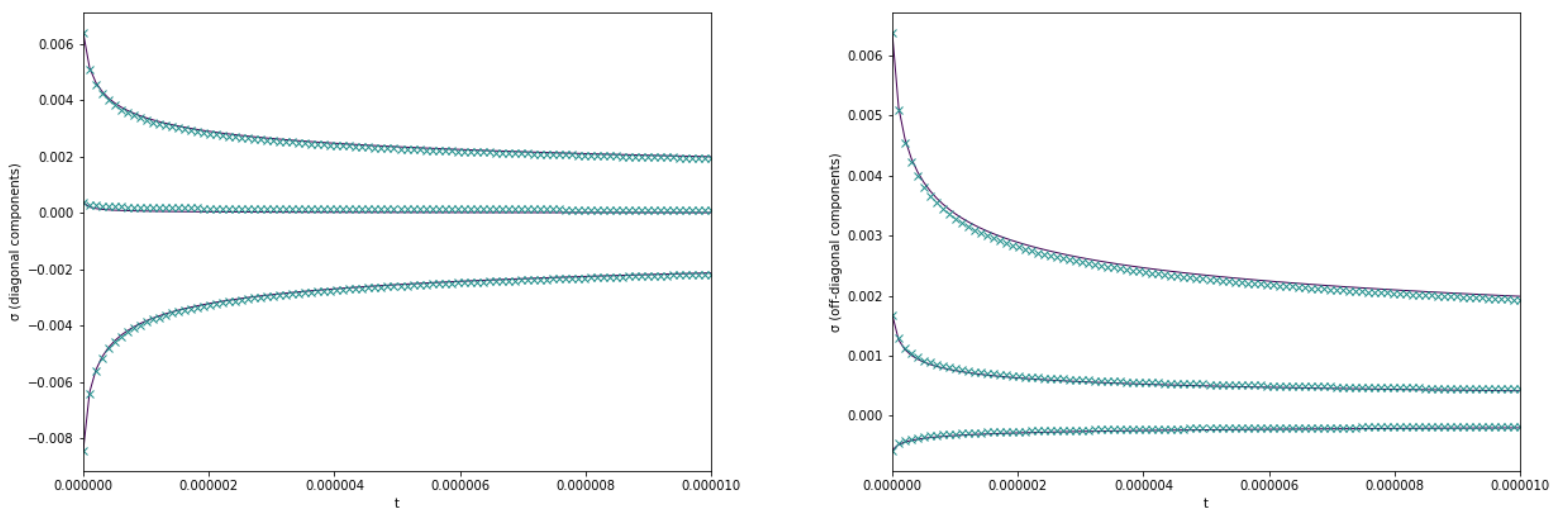

Figure 3: Stress tensor components during the Strain Relaxation Test: approximate analytical solution (crosses) and numerical ODE solution (solid line) 


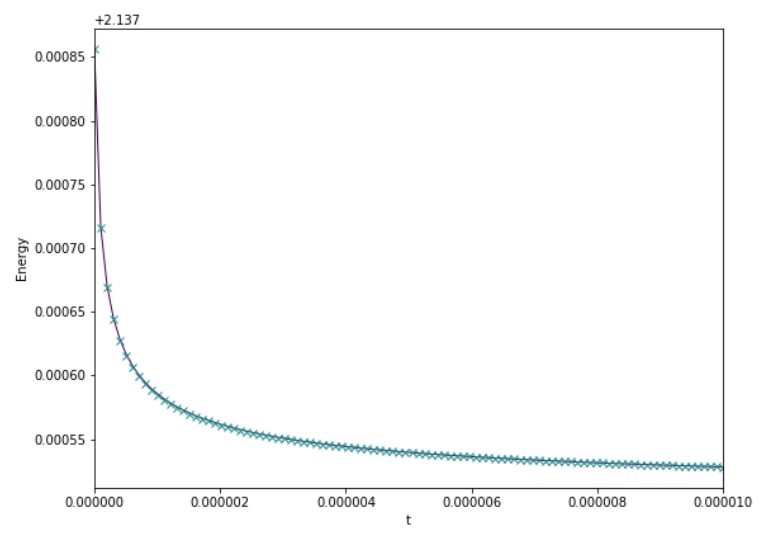

Figure 4: Total energy during the Strain Relaxation Test: approximate analytical solution (crosses) and numerical ODE solution (solid line)
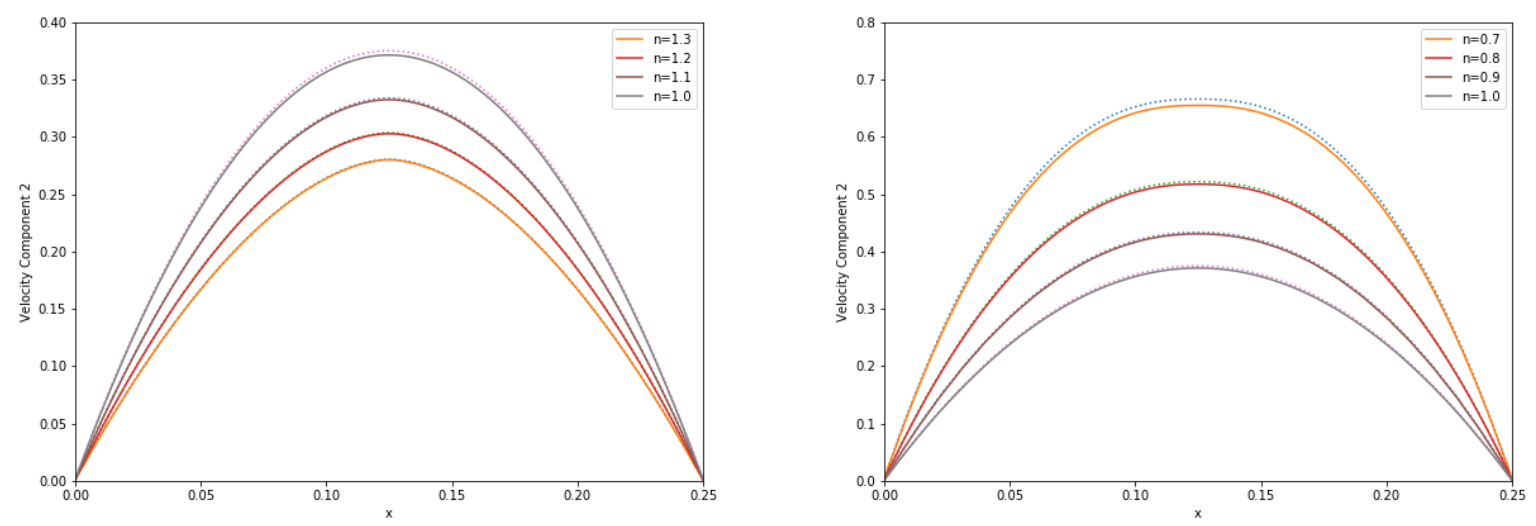

Figure 5: Velocity profiles for different dilatants (left) and pseudoplastics (right), in steady Poiseuille flow 

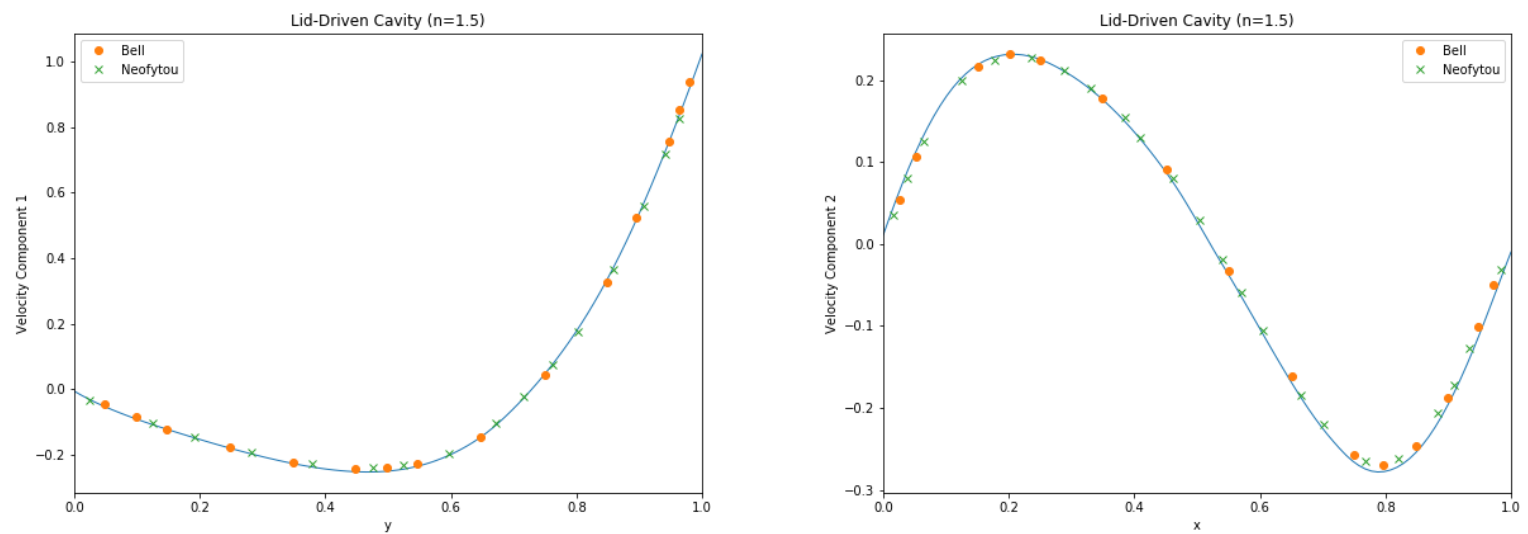

Figure 6: Velocity profiles for the Lid-Driven Cavity Test under our new formulation (solid line), for a dilatant with $\mathrm{n}=1.5$. Slices are taken through the center of the domain, in both axes, and compared with those of Bell and Surana [3] and Neofytou [19.

The test consists of a square grid, with one side at a constant velocity of 1 , and the other three stationary, with no-slip boundary conditions imposed. The fluid obeys an ideal gas EOS with $\gamma=1.4$ and $c_{s}=1$. It obeys a viscosity power law with $K=10^{-2}$, for various $n$. It is initially at rest, with $\rho=1, p=1, A=I$.

The grid is chosen to have size $100 \times 100$. A third order WENO method is used, with a CFL number of 0.5 .

Figure 6 on page 20 and Figure 7 on page 21 show the results of running the system to steady state, for $n=1.5$ and $n=0.5$, respectively. The results are compared with those of Bell and Surana [3] and Neofytou [19]. As can be seen, there is very good agreement for the case $n=1.5$, with the split solver performing slightly less well for the case $n=0.5$. The $2 \mathrm{D}$ streamline plots found in Figure 8 on page 21 take the characteristic forms found in the aforementioned literature.

\subsection{Elastoplastic Piston}

We now demonstrate the ability of our new numerical scheme to deal with problems involving elastoplastic materials. This test is taken from Peshkov et al. [20, with exact solutions found in Maire et al. [16].

In this test, a piston with speed $20 \mathrm{~ms}^{-1}$ is driven into copper initially at rest. An elastic shock wave develops, followed by a plastic shock wave. The following parameters were used: $\rho_{0}=8930, c_{s}=2244, \sigma_{0}=$ $9 \times 10^{7}, \tau_{0}=1$. The shock Mie-Gruneisen EOS is used for the internal energy, with $p_{0}=0, c_{0}=3940, \Gamma_{0}=$ $2, s=1.48$. 400 grid cells were used, with a third order WENO method, and a CFL number of 0.7.

Figure 9 on page 22 and Figure 10 on page 22 demonstrate the results using the split solver for various values of $n$. These results are compared with the exact solution to the problem under ideal plasticity (to which the former results should converge as $n \rightarrow \infty)$. The split solver is able to cope with larger values of $n$ than those that have been presented in Peshkov et al. [20]. The results here are correspondingly closer to the ideal plasticity solution that they approximate, than those found in the aforementioned paper.

\subsection{Cylindrical Shock}

The purpose of this test is to demonstrate the efficacy of the split solver in multidimensional elastoplastic problems. 

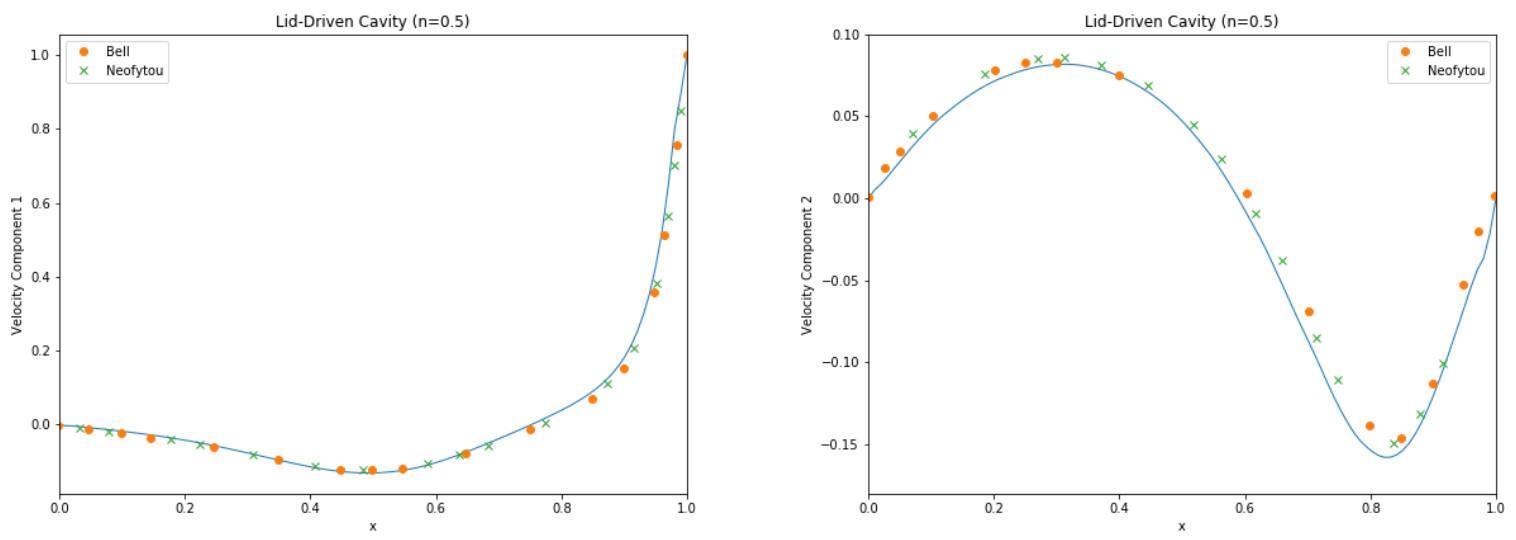

Figure 7: Velocity profiles for the Lid-Driven Cavity Test under our new formulation (solid line), for a pseudoplastic with $\mathrm{n}=0.5$. Slices are taken through the center of the domain, in both axes, and compared with those of Bell and Surana 3$]$ and Neofytou 19 .
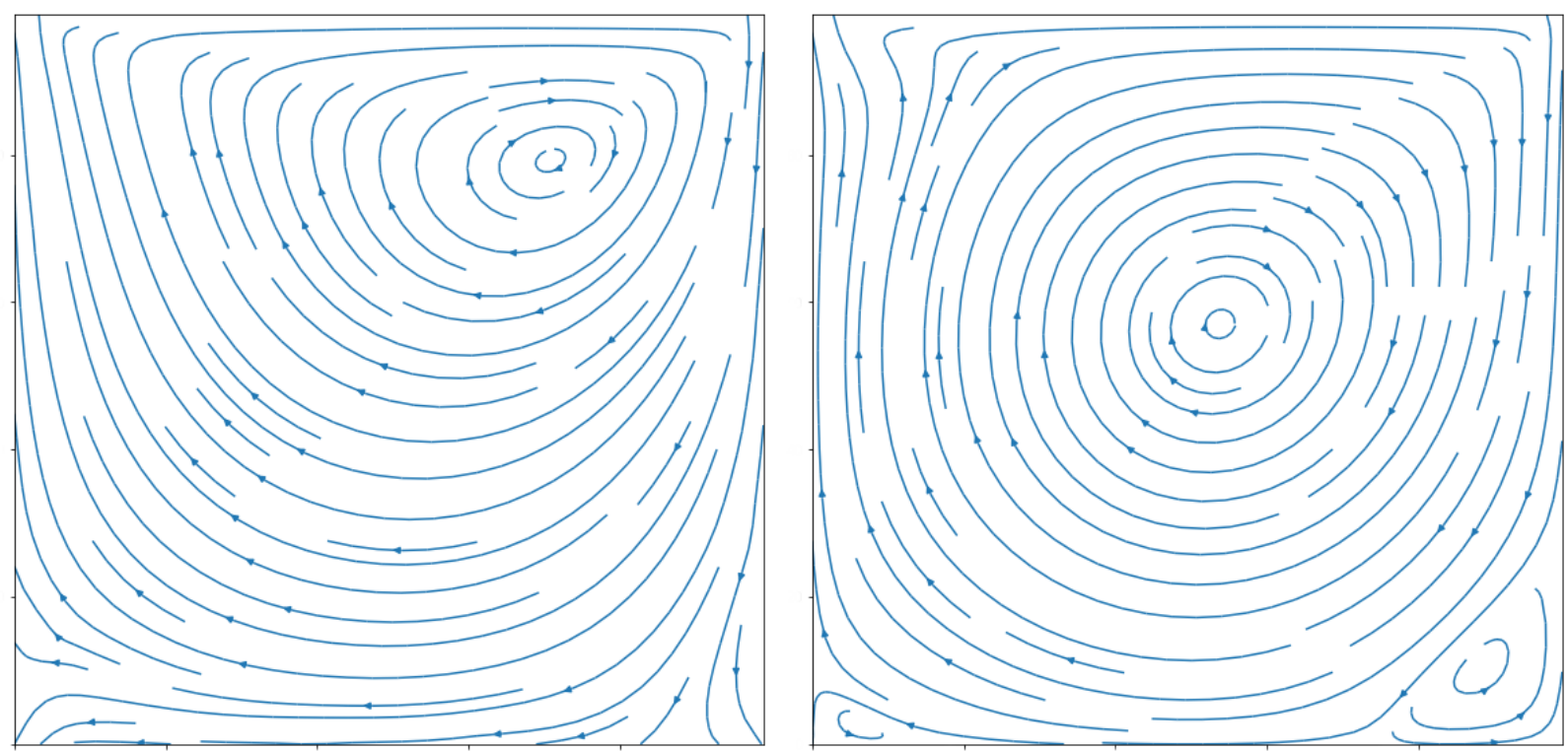

Figure 8: Streamplots for the Lid-Driven Cavity Test, for a pseudoplastic with $\mathrm{n}=0.5$ (left) and a dilatant with $\mathrm{n}=1.5$ (right) 

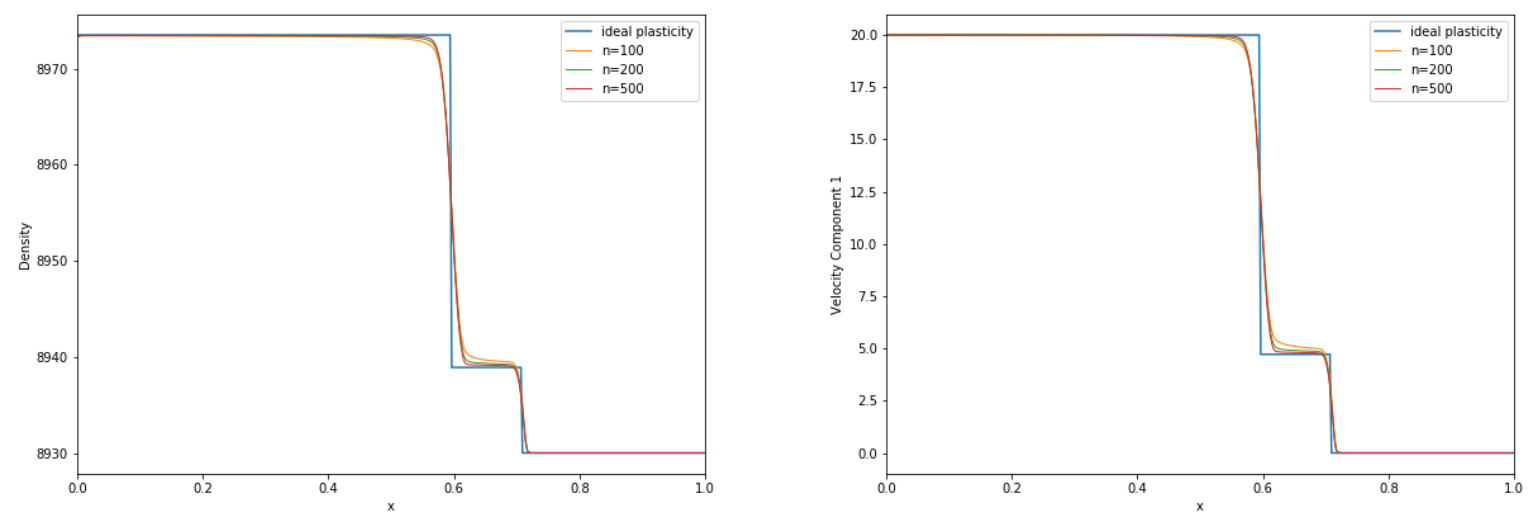

Figure 9: Density and velocity in the elastoplastic piston test, for various values of power-law parameter $n$
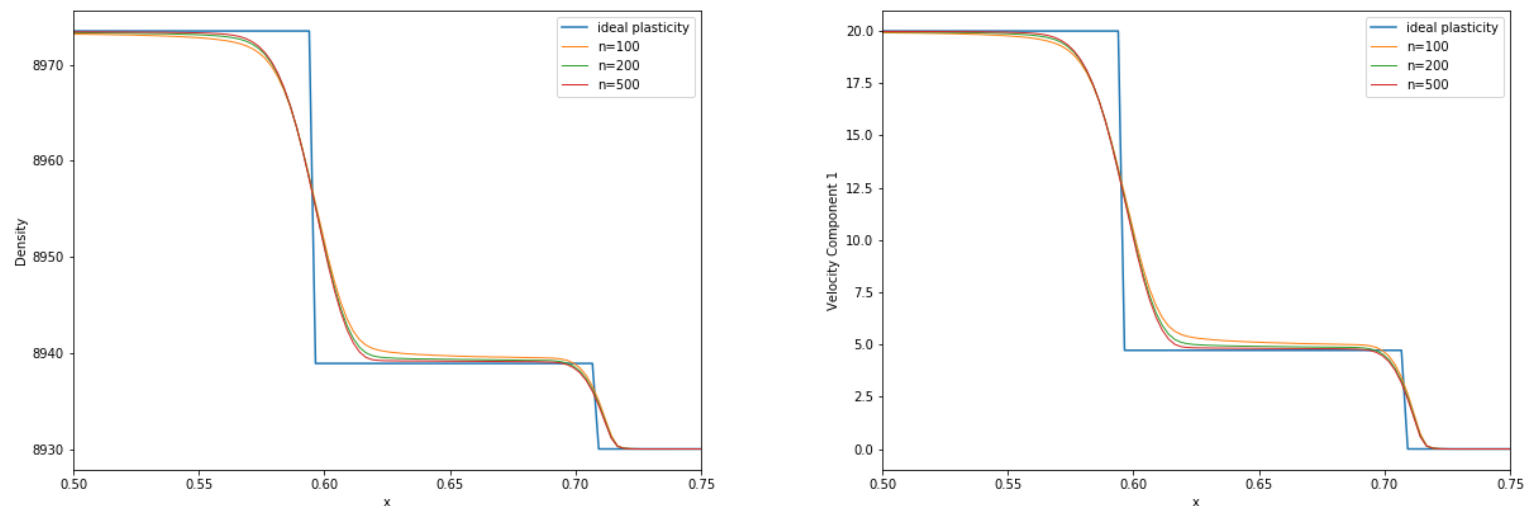

Figure 10: Zoom view of density and velocity in the elastoplastic piston test, for various values of power-law parameter $n$ 
This test is taken from Barton et al. 22. It consists of a slab of copper, occupying the domain $[0,20]^{2}$, initially at rest. The region $r \leq 2$ is at ambient conditions, with zero pressure. The region $r>2$ is at raised pressure $10^{10}$ and temperature 600 .

The simulation is run to time $t=10^{-5}$, on a grid of shape $500 \times 500$. A fourth order WENO scheme is used, with a CFL number of 0.8 . The resulting radial density, velocity, stress tensor, and temperature profiles are given in Figure 11 on page 24, Figure 12 on page 24, Figure 13 on page 25, Figure 14 on page 25, and 2D heatmaps for density and speed are given in Figure 15 on page 28

The results are compared with those of the 1D radially-symmetric scheme found in Barton et al. [2, which are in turn compared with the $2 \mathrm{D}$ results from the same publication. As can be seen, the $2 \mathrm{D}$ results computed using the new split solver for the GPR model more closely match the 1D radially-symmetric results than the $2 \mathrm{D}$ results from the aforementioned publication, with the spikes in both variables around $r=2$ and the wave around $r=6$ being more accurately resolved. Additionally, the temperature jump around $r=2$ is more sharply resolved.

\section{Conclusions}

In summary, a formulation for modeling power-law dilatants and pseudoplastics under the GPR model has been presented. A new numerical method - based on an operator splitting, combined with some analytical results - has also been presented for solving this version of the GPR model, and this numerical method has been applied also to the case of elastoplastic solids under a power-law plasticity model. It has been demonstrated through numerical simulation that the modified GPR formulation is able to accurately describe the evolution of non-Newtonian fluids, and the new numerical scheme has been shown to be an effective method by which to solve this system, and the existing corresponding system for elastoplastic solids.

Under circumstances in which the flow is compressed heavily in one direction relative to the other directions, it should be noted that the linearization assumption (53) used to derive the approximate analytical solver may break down. As discussed in Jackson [14, this is due to the fact that one of the singular values of the distortion tensor will be much larger than the others, and the mean of the squares of the singular values will be distant to the geometric mean. The subsequent linearization of the ODE governing the mean of the singular values will then fail. It should be noted that none of the situations covered in this study presented problems for the approximate analytical solver, and situations which may be problematic are in some sense unusual. In any case, a stiff ODE solver can be used to solve the systems 22ad, 22b if necessary, and so this method is still very much usable in these situations, albeit slightly slower.

As detailed in Leveque and Yee [15, solvers based on a temporal splitting suffer from a lack of spatial resolution in evaluating the source terms. Thus, it should be noted that the operator splitting method presented here may suffer from the incorrect speed of propagation of discontinuities on regular, structured grids. This issue can be rectified, however, by the use of some form of shock tracking or mesh refinement, as noted in the cited paper. Dumbser et al. [5] note that operator splitting-based methods can result in schemes that are neither well-balanced, nor asymptotically consistent. The extent to which these two conditions are violated by this method - and the severity in practice of any potential violation - is a topic of further research.

It should be noted that the new numerical scheme presented in this study is trivially parallelizable on a cell-wise basis. Thus, given a large number of computational cores, deficiencies in this method in terms of its order of accuracy may be overcome by utilizing a larger number of computational cells and cores. The number of grid cells that can be used scales roughly linearly with number of cores, at constant time per iteration. 

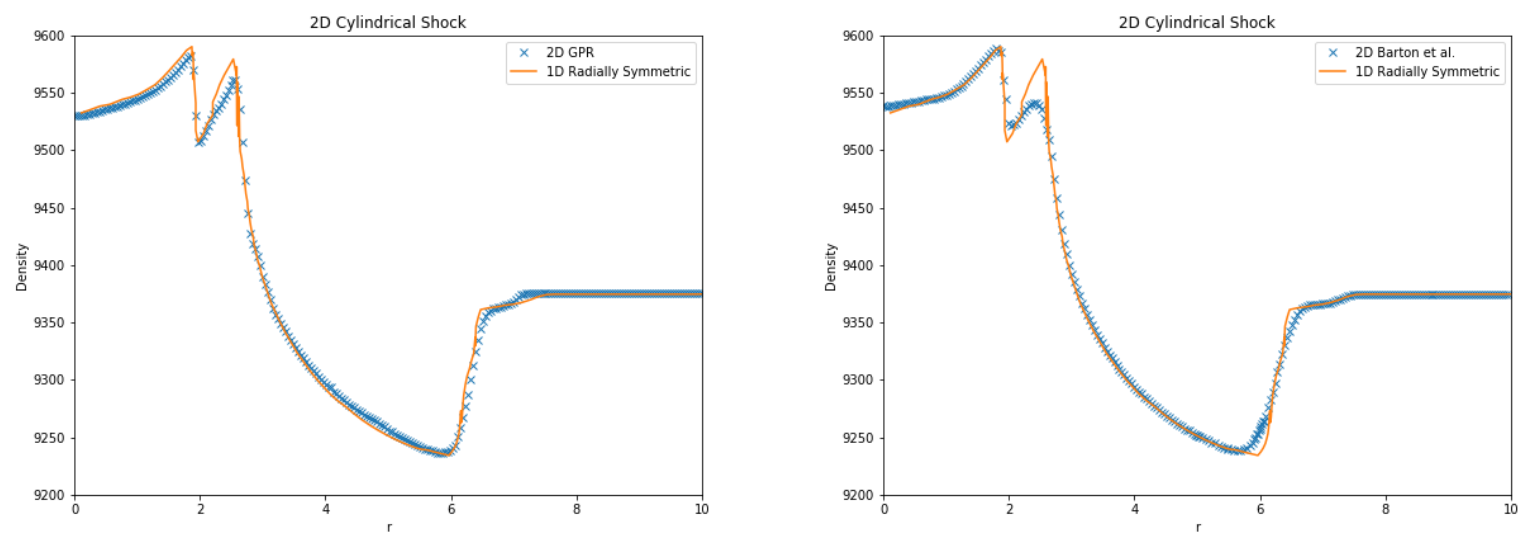

Figure 11: 1D density profiles for the 2D Cylindrical Shock Test, comparing the GPR model with split solver (left) to the results from Barton et al. 2] (right)
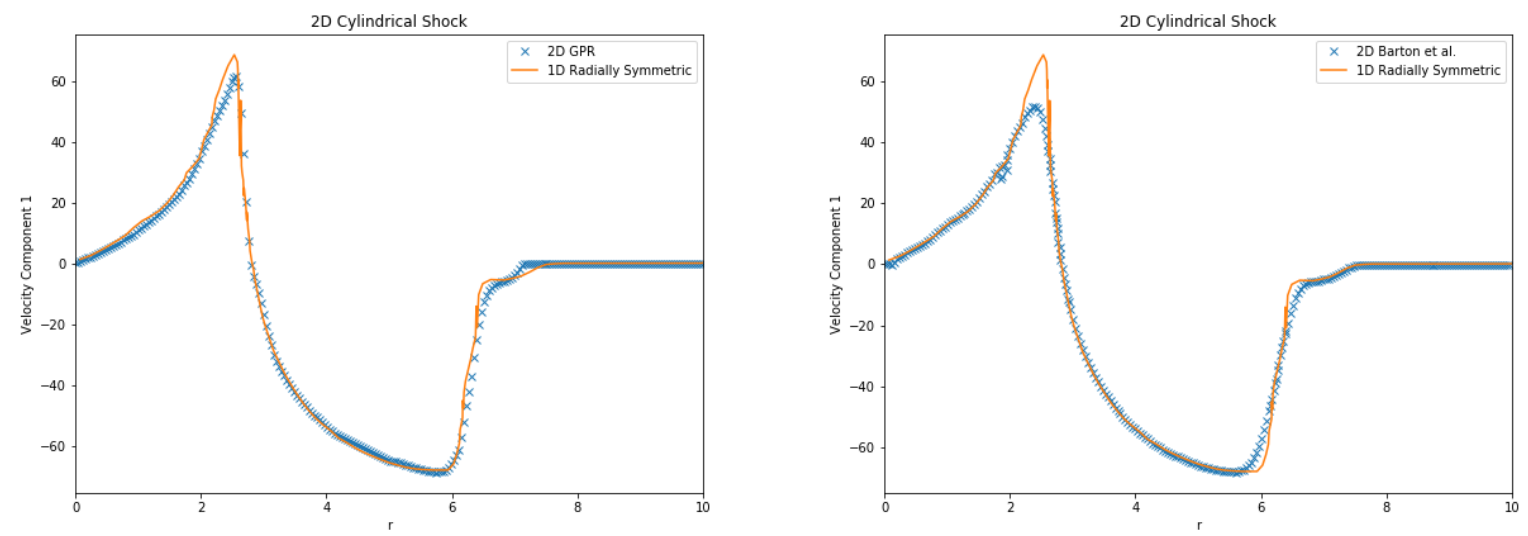

Figure 12: 1D velocity profiles for the 2D Cylindrical Shock Test, comparing the GPR model with split solver (left) to the results from Barton et al. [2] (right) 

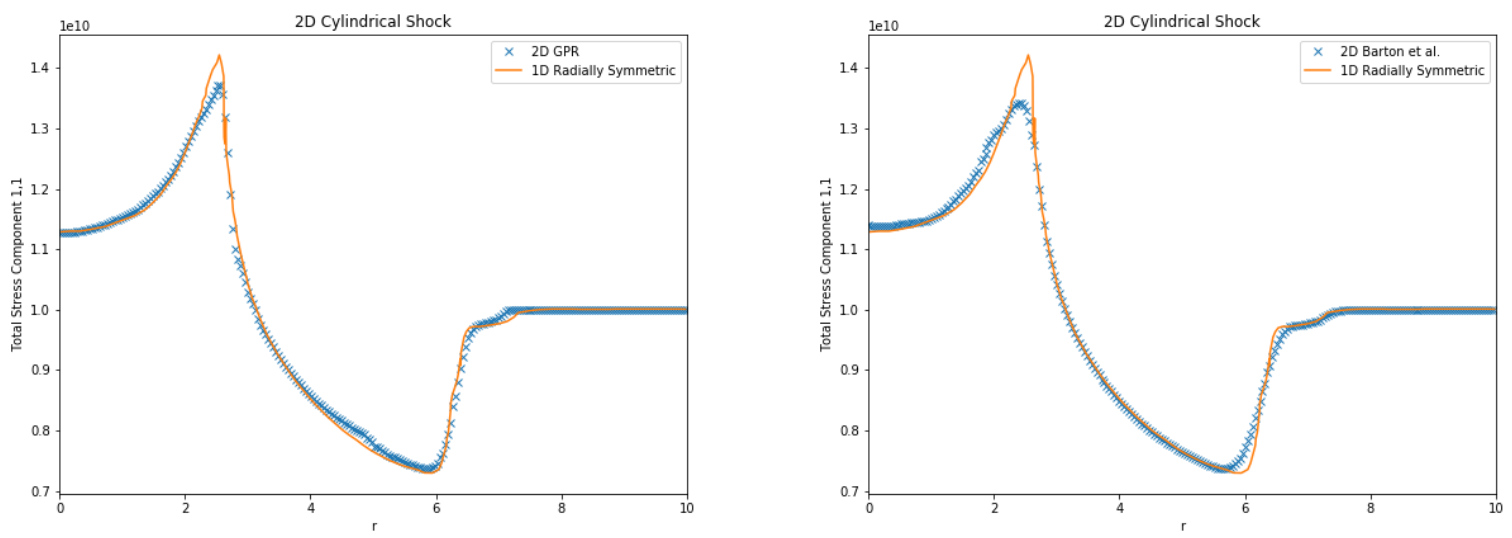

Figure 13: 1D stress tensor profiles for the 2D Cylindrical Shock Test, comparing the GPR model with split solver (left) to the results from Barton et al. 2] (right)
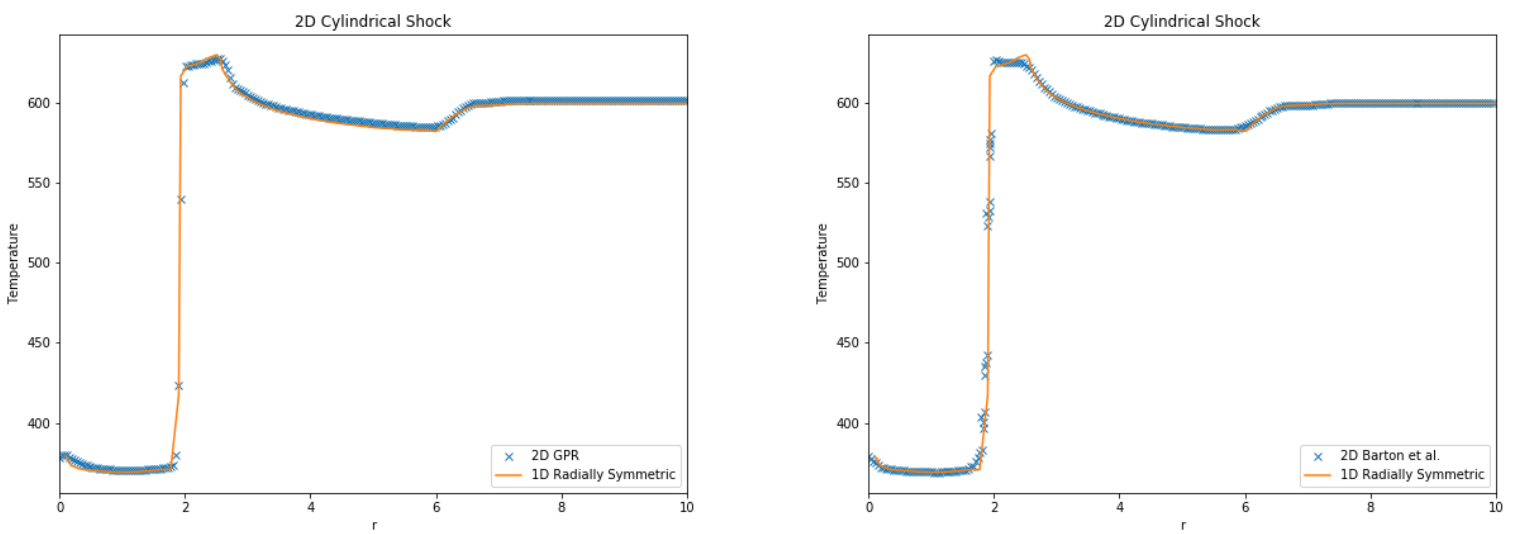

Figure 14: 1D temperature profiles for the 2D Cylindrical Shock Test, comparing the GPR model with split solver (left) to the results from Barton et al. 2] (right) 


\section{References}

\section{References}

[1] Barton, P. T., Drikakis, D., Romenski, E., Titarev, V. a., 2009. Exact and approximate solutions of Riemann problems in non-linear elasticity. Journal of Computational Physics 228 (18), 7046-7068.

[2] Barton, P. T., Drikakis, D., Romenski, E. I., 2011. An Eulerian finite-volume scheme for large elastoplastic deformations in solids. International Journal for Numerical Methods in Engineering 81, 453-484.

[3] Bell, B. C., Surana, K. S., Jan. 1994. p-version least squares finite element formulation for two-dimensional, incompressible, non-Newtonian isothermal and non-isothermal fluid flow. International Journal for Numerical Methods in Fluids 18 (2), 127-162.

[4] Boscheri, W., Dumbser, M., Loubere, R., 2016. Cell centered direct Arbitrary-Lagrangian-Eulerian ADER-WENO finite volume schemes for nonlinear hyperelasticity. Computers and Fluids 134-135, 111-129.

[5] Dumbser, M., Enaux, C., Toro, E. F., 2008. Finite volume schemes of very high order of accuracy for stiff hyperbolic balance laws. Journal of Computational Physics 227 (8), 3971-4001.

[6] Dumbser, M., Peshkov, I., Romenski, E., Zanotti, O., 2015. High order ADER schemes for a unified first order hyperbolic formulation of continuum mechanics: viscous heat-conducting fluids and elastic solids. Journal of Computational Physics 314, 824-862.

[7] Dumbser, M., Peshkov, I., Romenski, E., Zanotti, O., 2016. High order ADER schemes for a unified first order hyperbolic formulation of Newtonian continuum mechanics coupled with electro-dynamics.

[8] Dumbser, M., Toro, E. F., 2011. On universal Osher-type schemes for general nonlinear hyperbolic conservation laws. Communications in Computational Physics 10 (3), 635-671.

[9] Dumbser, M., Toro, E. F., 2011. A simple extension of the Osher Riemann solver to non-conservative hyperbolic systems. Journal of Scientific Computing 48 (1-3), 70-88.

[10] Dumbser, M., Zanotti, O., Hidalgo, A., Balsara, D. S., 2013. ADER-WENO finite volume schemes with space-time adaptive mesh refinement. Journal of Computational Physics 248, 257-286.

[11] Ferras, L. L., Nobrega, J. M., Pinho, F. T., 2012. Analytical solutions for Newtonian and inelastic non-Newtonian flows with wall slip. Journal of Non-Newtonian Fluid Mechanics 175-176, 76-88.

[12] Frenkel, J., 1947. Kinetic Theory of Liquids. Oxford University Press.

[13] Giles, M. B., 2008. An extended collection of matrix derivative results for forward and reverse mode algorithmic differentiation. Tech. Rep. 9783540689355. URL http://eprints.maths.ox.ac.uk/1079/

[14] Jackson, H., 2017. A Fast Numerical Scheme for the Godunov-Peshkov-Romenski Model of Continuum Mechanics. Journal of Computational Physics 348, 514-533. URL http://dx.doi.org/10.1016/j.jcp.2017.07.055

[15] Leveque, R., Yee, H., 1990. A Study of Numerical Methods for Hyperbolic Conservation Laws with Stiff Source Terms. Journal of Computational Physics 86, 187-210.

[16] Maire, P. H., Abgrall, R., Breil, J., Loubere, R., Rebourcet, B., 2013. A nominally second-order cell-centered Lagrangian scheme for simulating elastic-plastic flows on two-dimensional unstructured grids. Journal of Computational Physics 235 , $626-665$.

[17] Malyshev, A. N., Romenskii, E. I., 1986. Hyperbolic equations for heat transfer. Global solvability of the Cauchy problem. Siberian Mathematical Journal 27 (5), 734-740.

[18] McAdams, A., Selle, A., Tamstorf, R., Teran, J., Sifakis, E., 2011. Computing the Singular Value Decomposition of 3 x 3 matrices with minimal branching and elementary floating point operations. University of Wisconsin Madison.

[19] Neofytou, P., May 2005. A 3rd order upwind finite volume method for generalised Newtonian fluid flows. Advances in Engineering Software 36, 664-680.

URL https://dl .acm.org/citation.cfm?id=1276040.1276043

[20] Peshkov, I., Boscheri, W., LoubǍre, R., Romenski, E., Dumbser, M., Jun. 2018. Theoretical and numerical comparison of hyperelastic and hypoelastic formulations for Eulerian non-linear elastoplasticity. arXiv:1806.00706 [physics]ArXiv: 1806.00706 . URL http://arxiv.org/abs/1806.00706

[21] Peshkov, I., Romenski, E., 2016. A hyperbolic model for viscous Newtonian flows. Continuum Mechanics and Thermodynamics 28 (1-2), 85-104.

[22] Peshkov, I., Romenski, E., Dumbser, M., 2017. A unified hyperbolic formulation for viscous fluids and elastoplastic solids. URL http://arxiv.org/abs/1705.02151

[23] Peshkov, I., Romenski, E., Dumbser, M., Fambri, F., 2018. General Relativistic formulation of dissipative continuum mechanics.

[24] Romenski, E., Drikakis, D., Toro, E., 2010. Conservative models and numerical methods for compressible two-phase flow. Journal of Scientific Computing 42 (1), 68-95.

[25] Romenski, E., Resnyansky, A. D., Toro, E. F., 2007. Conservative hyperbolic model for compressible two-phase flow with different phase pressures and temperatures. Quarterly of applied mathematics 65(2) (2), 259-279.

[26] Romenski, E. I., 1989. Hyperbolic equations of Maxwell's nonlinear model of elastoplastic heat-conducting media. Siberian Mathematical Journal 30 (4), 606-625.

[27] Sverdrup, K., Nikiforakis, N., Almgren, A., Sep. 2018. Highly parallelisable simulations of time-dependent viscoplastic fluid flow with structured adaptive mesh refinement. Physics of Fluids 30 (9), 093102. 
[28] Toro, E. F., 2009. Reimann Solvers and Numerical Methods for fluid dynamics. Vol. 40.

\section{Acknowledgments}

The authors acknowledge financial support from the EPSRC Centre for Doctoral Training in Computational Methods for Materials Science under grant EP/L015552/1. 

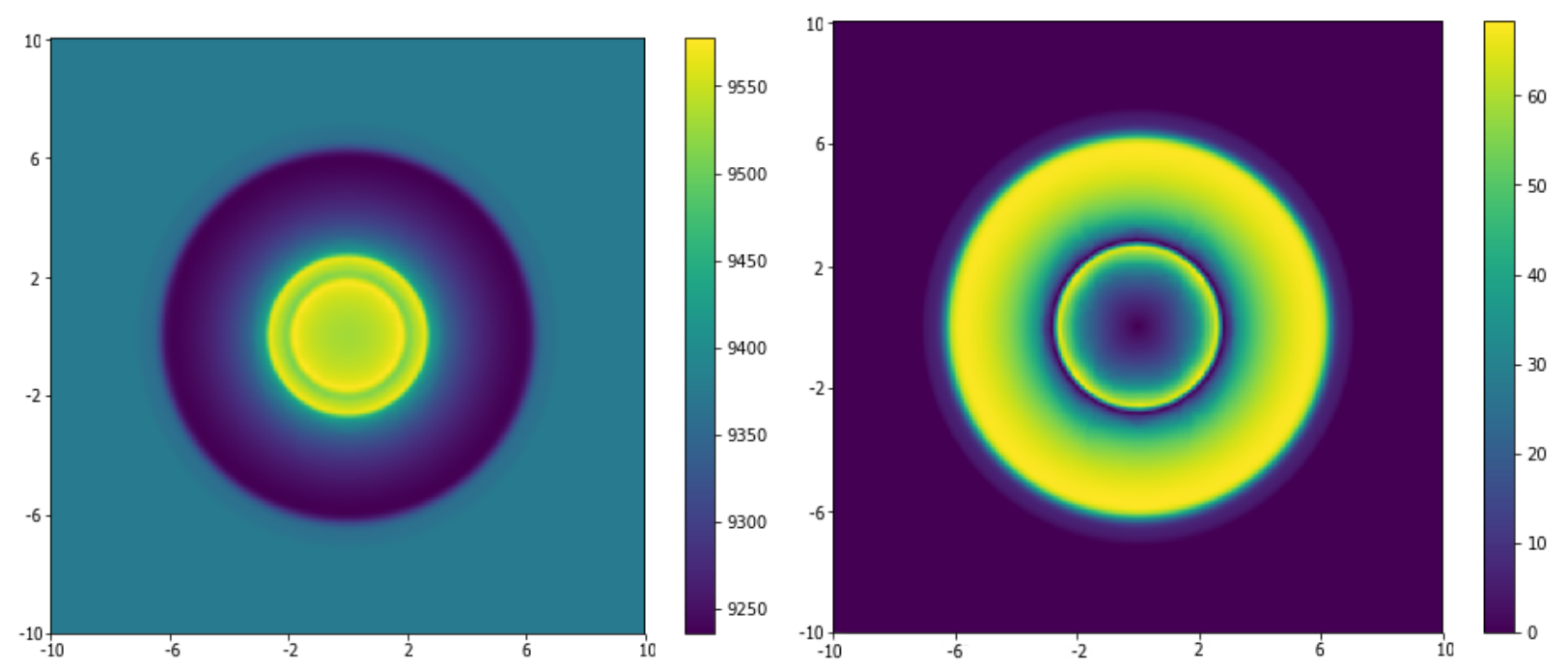

Figure 15: 2D plots of density and speed for the Cylindrical Shock Test 